\title{
Healthy and Osteoarthritic Synovial Fibroblasts Produce a Disintegrin and Metalloproteinase with Thrombospondin Motifs 4, 5, 7, and 12
}

\section{Induction by IL-1 $\beta$ and Fibronectin and Contribution to Cartilage Damage}

Selene Pérez-García, ${ }^{*}$ Irene Gutiérrez-Cañas, ${ }^{*}$ Iria V. Seoane, ${ }^{*}$ Julián Fernández, ${ }^{\dagger}$ Mario Mellado, ${ }^{\ddagger}$ Javier Leceta, ${ }^{*}$ Laura Tío, Raúl Villanueva-Romero, ${ }^{*}$ Yasmina Juarranz, ${ }^{*}$ and Rosa P. Gomariz*

From the Department of Cell Biology, ${ }^{*}$ Faculty of Biology, Complutense University of Madrid, Madrid; the Traumatology Service, ${ }^{\dagger}$ Hospital Universitario de La Princesa, Medical Research Institute, Madrid; the Department of Immunology and Oncology, ${ }^{\ddagger}$ Centro Nacional de Biotecnología, Consejo Superior de Investigaciones Cientificas (CSIC), Madrid; and the Cellular Inflammation and Cartilage Research Group, ${ }^{\S}$ Hospital del Mar Research Institute (IMIM), Barcelona, Spain

\author{
Accepted for publication \\ May 23, 2016. \\ Address correspondence to \\ Rosa P. Gomariz, Ph.D., \\ Department of Cell Biology, \\ Faculty of Biology, Complu- \\ tense University, C/ José Anto- \\ nio Novais $\mathrm{n}^{\circ} 2$, Ciudad \\ Universitaria, 28040 Madrid, \\ Spain. E-mail: gomariz@bio. \\ ucm.es.
}

\begin{abstract}
Current description of osteoarthritis includes the involvement of synovial inflammation. Studies contributing to understanding the mechanisms of cross-talk and feedback among the joint tissues could be relevant to the development of therapies that block disease progression. During osteoarthritis, synovial fibroblasts exposed to anomalous mechanical forces and an inflammatory microenvironment release factors such as a disintegrin and metalloproteinase with thrombospondin motifs (ADAMTS) metalloproteinases that mediate tissue damage and perpetuate inflammation. We therefore studied the production of ADAMTS by synovial fibroblasts and their contribution to cartilage degradation. Moreover, we analyzed the implication of two mediators present in the osteoarthritis joint, IL-1 $\beta$ as proinflammatory cytokine, and $45-k$ Da fibronectin fragments as products of matrix degradation. We reported that synovial fibroblasts constitutively express and release ADAMTS 4, 5, 7, and 12. Despite the contribution of both mediators to the stimulation of Runx2 and Wnt/ $\beta$-catenin signaling pathways, as well as to ADAMTS expression, promoting the degradation of aggrecan and cartilage oligomeric matrix protein from cartilage, fibronectin fragments rather than IL-1 $\beta$ played the major pathological role in osteoarthritis, contributing to the maintenance of the disease. Moreover, higher levels of ADAMTS 4 and 7 and a specific regulation of ADAMTS-12 were observed in osteoarthritis, suggesting them as new potential therapeutic targets. Therefore, synovial fibroblasts provide the biochemical tools to the chronicity and destruction of the osteoarthritic joints. (Am J Pathol 2016, 186: 2449-2461; http://dx.doi.org/10.1016/j.ajpath.2016.05.017)
\end{abstract}

Osteoarthritis (OA), one of the leading causes of substantial physical and psychological disability worldwide, is a complex disease with a prevalence of $>70 \%$ in the population $>55$ years. ${ }^{1,2}$ Current description of OA includes not only the remodeling of articular cartilage and adjacent bone, but also the involvement of synovial inflammation, which is characterized by thickening of synovium or, indirectly, by joint effusion. Synovial membrane inflammation and proliferation
Supported by Instituto de Salud Carlos III, Spain, cofinanced by FEDER, European Union: RETICS program, Red de Investigación en Inflamación y Enfermedades Reumáticas (RD12/0009/0002), the project (PI12/00758; S.P.-G.), and Comunidad de Madrid/FEDER grant (RAPHYME program, S2010/BMD2350; R.V.-R.).

R.P.G. and Y.J. contributed equally to this work.

Disclosures: None declared. 
in $\mathrm{OA}$ joints trigger the production of cytokines and proteinases that damage connective tissues, including the cartilage. ${ }^{3,4}$ In this sense, research on inhibitory mediators of synovial activation could identify ways to avoid the progressive cartilage degradation and functional impairment. The role of synovial fibroblasts (SFs) as active drivers of joint destruction in rheumatoid arthritis is well established, ${ }^{5}$ but their behavior in healthy subjects and OA patients is poorly understood. It has been described that OA-SF exposed to anomalous mechanical forces and to an inflammatory microenvironment, release factors such as a disintegrin and metalloproteinase with thrombospondin motifs (ADAMTS), that mediate tissue damage and perpetuate inflammation. ${ }^{2,6-9}$ Therefore, studies contributing to a better understanding of the cross-talk and feed-back mechanisms among the joint tissues could be relevant to the development of new therapies able to block disease progression.

Our aim was to elucidate the role of SF in the cartilage joint degradation in $\mathrm{OA}$ patients through the production of ADAMTS and to characterize these metalloproteinases in SF from healthy donors (HD). We have mapped the expression and function of aggrecanases ADAMTS 4 and 5, which degrade aggrecan, one of the main components of the cartilage extracellular matrix $(\mathrm{ECM})$ that facilitates cartilage to resist compression. ${ }^{10}$ We have also characterized ADAMTS 7 and 12, involved in destruction of cartilage oligomeric matrix protein (COMP), a noncollagenous component of the cartilage ECM that contributes to its assembly and to the cartilage integrity. ${ }^{11}$ Moreover, we studied the physiopathological effect of two mediators present in OA joint microenvironment: the catabolic cytokine IL- $1 \beta$ and the $45-\mathrm{kDa}$ fibronectin fragments (Fn-fs) as products of cartilage ECM degradation. ${ }^{12-15}$ This study is the first to report the expression and release of ADAMTS 7 and 12 by SF from HD and OA patients, both constitutively and after IL- $1 \beta$ or Fn-fs stimulation. Besides, the capacity of SF to attach and degrade the cartilage ECM, generating glycosaminoglycans (GAGs) and releasing COMP, is also described. Finally, we study the activation of Runx 2 and $\beta$-catenin, two signaling pathways related to ADAMTS expression. ${ }^{16-18}$ Our study reports that SF activated by mediators present in the joint, such as a proinflammatory cytokines and Fn-fs, release ADAMTS, which contribute to the maintenance of cartilage destruction in osteoarthritic patients.

\section{Materials and Methods}

\section{Patients and Synovial Fibroblast Cultures}

Synovial tissue was obtained from 20 active OA patients (16 women and 4 men) aged between 48 and 87 years, at the time of knee prosthetic replacement surgery. Patients had advanced disease and were diagnosed with primary OA, excluding trauma, inflammatory disease, and other structural causes of secondary OA. Control samples from HD were obtained from four patients (two women and two men) aged between 35 and
72 years, at the time of knee arthroscopic evaluation. These patients were diagnosed with meniscopathy caused by trauma to the knee or sports injury, excluding inflammatory and rheumatic diseases. The study was performed according to the recommendations of the Declaration of Helsinki and approved by the Clinical Research Ethics Committee of the Hospital La Princesa (Madrid, Spain). All biopsy samples were obtained after subjects gave informed consent.

SF cultures were established by explant growth of synovial biopsies, cultured in Dulbecco's modified Eagle's medium (DMEM) with $25 \mathrm{mmol} / \mathrm{L}$ HEPES and $4.5 \mathrm{~g} / \mathrm{L}$ glucose, completed with $10 \%$ heat-inactivated fetal bovine serum (Lonza Ibérica S.A.U., Barcelona, Spain), 1\% L-glutamine, and $1 \%$ antibiotic-antimycotic (Invitrogen, Carlsbad, CA) at $37^{\circ} \mathrm{C}$ and $5 \% \mathrm{CO}_{2}$. After three passages, residual contamination by macrophages was avoided, previously assessed by flow cytometry analysis of SF with a purity of $95 \% .{ }^{19}$ Monocultures of SF were used for experiments until passage 8 . Despite the use of cells at varying passage numbers, all comparisons within a same experimentation were made on $\mathrm{SF}$ at an identical passage number and at $80 \%$ to $90 \%$ confluence.

For treatments, HD- and OA-SF were cultured in serumfree DMEM with $1 \%$ L-glutamine and $1 \%$ antibioticantimycotic, in the absence (untreated) or presence of the following agents: $10 \mathrm{ng} / \mathrm{mL}$ IL-1 $\beta$ (ImmunoTools, Friesoythe, Germany) or $10 \mathrm{nmol} / \mathrm{L}$ Fn-fs $45 \mathrm{kDa}$ (SigmaAldrich, St Louis, MO).

\section{RNA Extraction and Quantitative RT-PCR for ADAMTS Gene Expression}

SFs were seeded in $100-\mathrm{mm}$ petri dishes $\left(3 \times 10^{5}\right.$ cells per dish) and cultured in the absence or presence of $10 \mathrm{ng} / \mathrm{mL}$ IL$1 \beta$ or $10 \mathrm{nmol} / \mathrm{L} \mathrm{Fn}-\mathrm{fs} 45 \mathrm{kDa}$ for 24 hours. Total RNA was obtained using TriReagent (Sigma-Aldrich). Two microgram of RNA was reverse transcribed using a High Capacity cDNA Reverse Transcription Kit (Applied Biosystems, Foster City, CA). Semiquantitative real-time PCR analysis was performed using a TaqMan Gene Expression Master Mix with manufactured-predesigned primers and probes for $\beta$-actin (NM001101.3), ADAMTS-4 (NM005099.4), ADAMTS-5 (NM007038.3), ADAMTS-7 (NM014272.3), and ADAMTS-12 (NM030955.2) (Applied Biosystems). We normalized the target gene expression to the housekeeping gene, $\beta$-actin $\left(2^{-\Delta \mathrm{Ct}}\right)$. For relative quantification, results were presented as the relative expression with respect to the untreated condition using the formula $2^{-\Delta \Delta \mathrm{Ct}}$, as previously described. ${ }^{19}$

\section{Quantification of ADAMTS in Culture Supernatants}

SFs were seeded in 6 -well plates $\left(6 \times 10^{4}\right.$ cells per well $)$ and cultured in the absence or presence of $10 \mathrm{ng} / \mathrm{mL} \mathrm{IL-} 1 \beta$ or 10 $\mathrm{nmol} / \mathrm{L}$ Fn-fs $45 \mathrm{kDa}$, for 24 hours. Levels of ADAMTS were measured in the culture supernatants using commercial enzyme-linked immunosorbent assay (ELISA) kits for 
ADAMTS 4 and 5 (Cloud-Clone Corp., Houston, TX), and for ADAMTS 7 and 12 (MyBioSource, San Diego, CA).

\section{Aggrecanase Activity Assay}

SFs were seeded in 100 -mm petri dishes $\left(3 \times 10^{5}\right.$ cells per dish) and cultured in the absence or presence of $10 \mathrm{ng} / \mathrm{mL}$ IL-1 $\beta$ or $10 \mathrm{nmol} / \mathrm{L}$ Fn-fs $45 \mathrm{kDa}$, for 24 hours. Aggrecanase activity was measured in the SF culture supernatants using a Sensitive Aggrecanase Activity ELISA Kit (MD Bioproducts, Zürich, Switzerland), according to the manufacturer's instructions. Briefly, this assay consists of two modules. In the Aggrecanase Module, a modified interglobular domain (aggrecan-IGD-s) is digested with aggrecanases, and its proteolytic cleavage releases an aggrecan peptide (ARGSVIL-peptide-s), which is then quantified with antibodies in the ELISA Module.

\section{Immunocytochemistry}

SFs were seeded on glass coverslips $\left(2.5 \times 10^{4}\right.$ cells per glass), fixed with paraformaldehyde, and permeabilized with Tween-20 in phosphate-buffered saline. Cells were blocked with phosphate-buffered saline containing donkey serum and incubated with rabbit polyclonal anti-human antibodies for ADAMTS 4, 5, 7, or 12 (Sigma-Aldrich). After washing, cells were incubated with AlexaFluor 488 donkey anti-rabbit IgG antibody (Invitrogen). Coverslips were counterstained with Hoechst. Background fluorescence was reduced with Sudan Black in ethanol. Negative controls were performed in the absence of primary antibodies (data not shown). Fluorescence was examined using an Olympus BX51 microscope with DP72 camera model (objective 40×).

\section{Runx2 Assay}

SFs were seeded in 150 -mm petri dishes $\left(8 \times 10^{5}\right.$ cells per dish). A Nuclear Extract Kit (Active Motif, Rixensart, Belgium) was used for nuclear extracts preparation, and the protein content was measured with a QuantiPro BCA Assay Kit (Sigma-Aldrich). Cytoplasmic extracts obtained were stored at $-80^{\circ} \mathrm{C}$ for later use in the Western blots. Nuclear extracts ( $12 \mu \mathrm{g}$ per well) were added to a 96-well plate, and Runx2 activity was measured using a TransAM AML-3/ Runx2 kit (Active Motif). Time course of Runx2 activation after incubation with $10 \mathrm{ng} / \mathrm{mL}$ IL-1 $\beta$ or $10 \mathrm{nmol} / \mathrm{L}$ Fn-fs 45 $\mathrm{kDa}$ was studied (data not shown), and the experiments were performed at 60 or 30 minutes of treatment, respectively.

\section{$\beta$-Catenin Assay}

To detect $\beta$-catenin levels, a $\beta$-catenin (Total) and a (Phospho) InstantOne ELISA kits were used (eBioscience, San Diego, CA) with SF cellular lysates. Briefly, SFs seeded in 100-mm petri dishes $\left(3 \times 10^{5}\right.$ cells per dish) were scraped into phosphate-buffered saline, centrifuged, and resuspended in the Cell Lysis Buffer Mix (eBioscience). Protein content was measured by QuantiPro BCA Assay Kit. Levels of $\beta$-catenin in the cellular lysates were measured after 60 minutes of treatment with $10 \mathrm{ng} / \mathrm{mL} \mathrm{IL-} 1 \beta$ or $10 \mathrm{nmol} / \mathrm{L}$ Fn-fs $45 \mathrm{kDa}$.

\section{Western Blots}

For the detection of ADAMTS, SFs were seeded in 100-mm dishes and cultured to confluence. Culture supernatants were collected. For protein purification and concentration, Amicon Ultra $0.5 \mathrm{~mL}$ centrifugal filters (Merck Millipore, Darmstadt, Germany) were used. For Runx 2 and $\beta$-catenin, the cytoplasmic extracts previously obtained were used, and protein content was measured by QuantiPro BCA Assay Kit. Cytoplasmic extracts $(15 \mu \mathrm{g} / \mathrm{well})$ and culture supernatants were subjected to SDS-PAGE and blotted on a polyvinylidene difluoride membrane (Bio-Rad Laboratories, Hercules, CA).

Membranes were blocked with Tris-buffered saline containing bovine serum albumin and Tween-20, and incubated with mouse monoclonal anti-human ADAMTS-4, ADAMTS5 (R\&D Systems), Runx2, or $\beta$-catenin (Santa Cruz Biotechnology) antibodies, or rabbit polyclonal anti-human ADAMTS-7 or ADAMTS-12 antibodies (Abcam, Cambridge, UK). Appropriate horseradish peroxidase-conjugated secondary antibodies were applied and detected by Western blot Luminol Reagent (Santa Cruz Biotechnology). For Runx2 and $\beta$-catenin, we used $\beta$-actin as a loading control. Protein bands were scanned and quantified with the Bio-Rad Quantity One program.

\section{Blockade Experiments}

For blockade experiments, HD- and OA-SF were seeded in 100 -mm dishes $\left(3 \times 10^{5}\right.$ cells per dish $)$ and cultured in serum-free DMEM with $1 \%$ L-glutamine and $1 \%$ antibioticantimycotic, in the absence or presence of $10 \mu \mathrm{mol} / \mathrm{L}$ of mitogen-activated protein kinase (MAPK)/extracellular signal-regulated kinase (ERK) kinases inhibitor, PD98059, consequently implicated in the inhibition of ERK; $10 \mu \mathrm{mol} /$ L of MAPK) p38 inhibitor, SB203580 (Calbiochem, EMD Biosciences, San Diego, CA), or $200 \mathrm{ng} / \mathrm{mL}$ of Wnt inhibitor, DDK-1 (R\&D Systems) for 1 hour. These treatments were followed by stimulation with $10 \mathrm{ng} / \mathrm{mL} \mathrm{IL}-1 \beta$ or $10 \mathrm{nmol} / \mathrm{L}$ Fn-fs $45 \mathrm{kDa}$, for 24 hours. Total RNA was obtained, and quantitative RT-PCR for ADAMTS 4 and 5 was performed as previously described. ${ }^{19}$

\section{GAGs and COMP Assays in Cartilage-SF Co-Cultures}

Release of GAG and COMP from cartilage was measured in culture supernatants from wells containing co-cultures of SF over cartilage explants. ${ }^{20}$ OA human cartilages were obtained from three patients undergoing total hip arthroplasty from Hospital del Mar (Barcelona, Spain). Fixed diameter $(6 \mathrm{~mm})$ and height $(2 \mathrm{~mm})$ sections were collected from cartilage areas without macroscopic signs of OA. Samples 
were frozen at $-80^{\circ} \mathrm{C}$ and stored until testing. One explant per well was attached to a 24-well plate. HD- or OA-SF were added drop-wise on top of the cartilage surface $\left(2 \times 10^{4} \mathrm{SF}\right.$ per explant). After 3 hours of incubation, wells were filled with DMEM in the absence or presence of $10 \mathrm{ng} /$ $\mathrm{mL}$ IL- $1 \beta$ or $10 \mathrm{nmol} / \mathrm{L}$ Fn-fs $45 \mathrm{kDa}$, and cultures were continued for 14 days. Culture supernatants were collected for detection of GAG and COMP, using a Blyscan Sulfated Glycosaminoglycan Assay (Biocolor Ltd County Antrim, Ireland, UK), and a Quantikine Human COMP Immunoassay (R\&D Systems, Abingdon, OX, UK), respectively. Frozen sections were prepared using a cryostat and stained with Alcian blue and Callejas's tricromic. Sections were observed using an Olympus BX51 microscope with DP72 camera model (objective $20 \times$ ).

\section{Statistical Analysis}

Data were analyzed using the GraphPad Prism software version 6 (Graphpad Software Inc., La Jolla, CA). Data were subjected to normality test (Kolmogórov-Smirnov test) and equal variance test (F-test). Statistical differences between sample groups were assessed using Student's two-tailed $t$-test or unpaired $t$-test with Welch's correction, in case of groups with different variances. $P<0.05$ was considered statistically significant. Results are presented as the means \pm SEM.

\section{Results}

SFs Express and Release ADAMTS 4, 5, 7, and 12 in HD and $\mathrm{OA}$ Patients

We explored the constitutive expression of ADAMTS in HDand OA-SF by quantitative RT-PCR (Figure 1). Patterns of constitutive ADAMTS gene expression were similar in untreated HD- and OA-SF. ADAMTS-5 was the most expressed

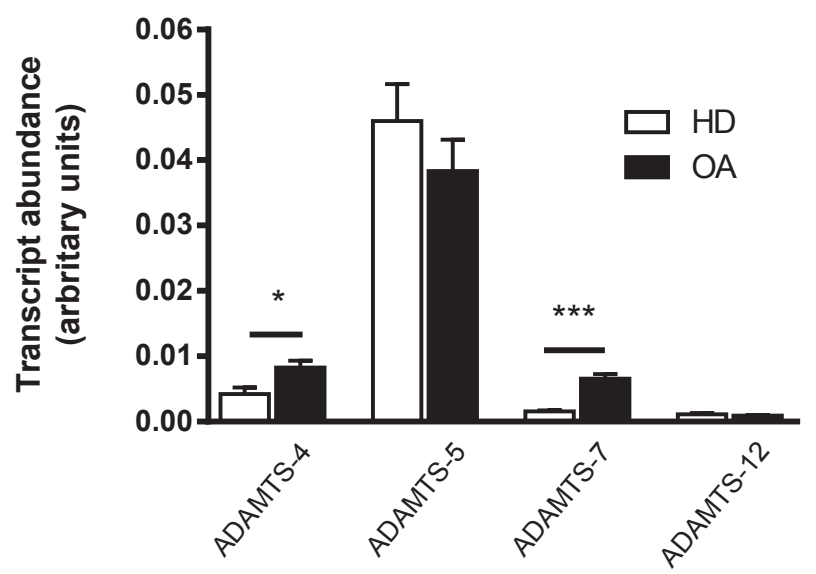

Figure 1 mRNA expression of ADAMTS in HD- and OA-SF. ADAMTS 4, 5, 7, and 12 mRNA expression was measured in untreated HD- and OA-SF by quantitative RT-PCR and normalized to $\beta$-actin using the formula $2^{-\Delta C \mathrm{Ct}}$ (described in Materials and Methods). Data are presented as means \pm SEM of triplicate determinations. $n=4(\mathrm{HD}) ; n=11(0 \mathrm{~A}) .{ }^{*} P<0.05$, ${ }^{* * *} P<0.001 \mathrm{HD}$ versus $\mathrm{OA}$. followed by ADAMTS 4, 7, and 12. ADAMTS-5 transcripts were nearly 50-fold higher than ADAMTS-12. Comparing HD- and OA-SF, ADAMTS 4 and 7 transcripts were twofold and fourfold higher in OA- than in HD-SF, respectively. However, ADAMTS 5 and 12 mRNA levels were similar.

ADAMTS protein expression was confirmed by immunocytochemistry. Untreated HD- and OA-SF displayed similar morphology and both showed cytoplasmic immunostaining for ADAMTS 4, 5, 7, and 12 (Figure 2). No staining was observed in isotype controls (data not shown).
HD
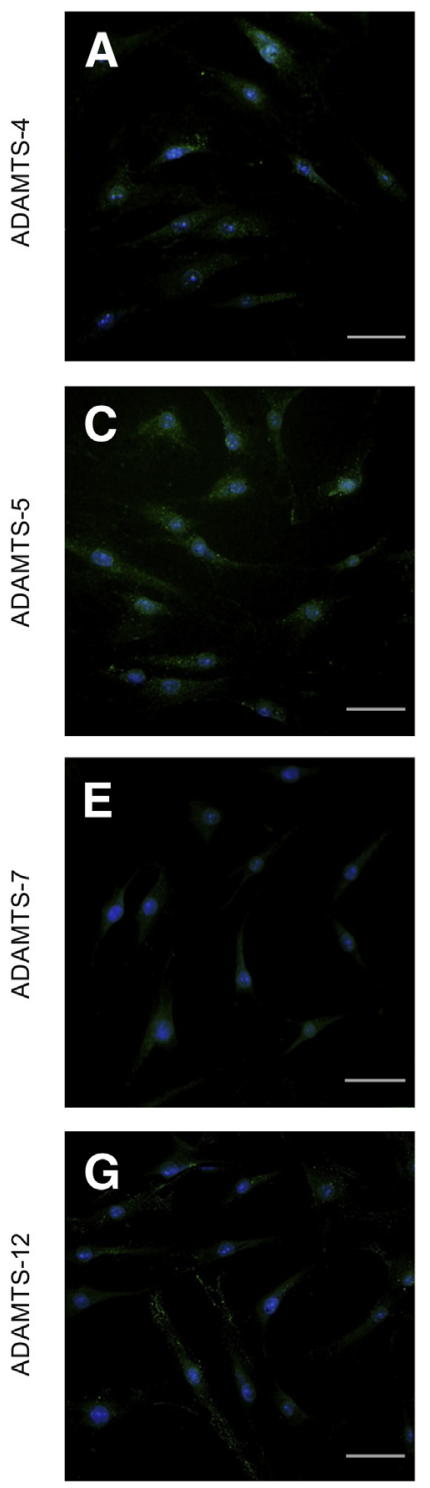

OA
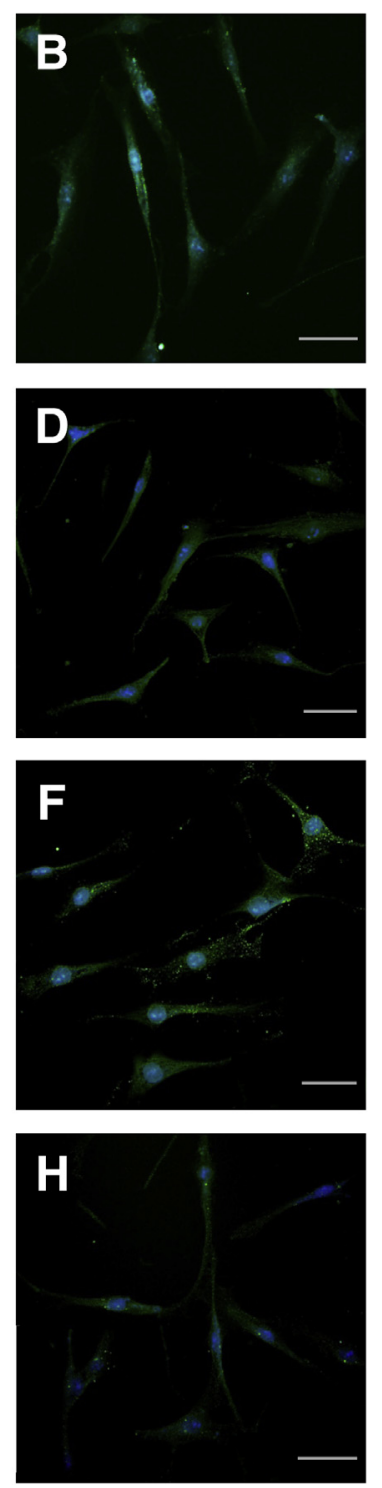

Figure 2 Immunocytochemistry of ADAMTS in HD- and OA-SF. Protein expression of ADAMTS-4 (A and B), ADAMTS-5 (C and $\mathbf{D})$, ADAMTS-7 ( $\mathbf{E}$ and F), and ADAMTS-12 ( $\mathbf{G}$ and $\mathbf{H}$ ) in untreated HD- and OA-SF was analyzed by immunofluorescence staining (green). Nuclei were counterstained with Hoeschst (blue). Immunostaining of the SF from one $\mathrm{HD}(\mathbf{A}, \mathbf{C}, \mathbf{E}$, and $\mathbf{G})$ and one $O A$ (B, D, F, and $\mathbf{H}$ ) patient; representative of three independent experiments. Fluorescence was examined on an Olympus BX51 microscope with DP72 camera mode (objective, $40 \times$ ). Scale bars $=50 \mu \mathrm{m}(\mathbf{A}-\mathbf{H}) . \mathrm{HD}$, healthy donors. 
ADAMTS were also evaluated in untreated SF culture supernatants by Western blot (Figure 3). We confirmed that all ADAMTS are released to the medium by HD- and OA-SF. Western blots for ADAMTS 4 and 5 (Figure 3, A and B) revealed bands corresponding to the active forms (between 48 and $74 \mathrm{kDa}$ ), and additional bands with a higher molecular weight. ADAMTS-7 and ADAMTS-12 Western blots (Figure 3, C and D) showed bands with the predicted molecular weight of the enzymes (between 114 and $201 \mathrm{kDa}$ ), and additional smaller bands.

\section{IL-1 $\beta$ and $45-k D a$ Fn-fs Enhance the Expression of ADAMTS- 4 and ADAMTS- 5 in SF}

Because SF has been suggested to represent an important source of aggrecanases within the joint mediating cartilage destruction, ${ }^{10}$ we next studied the effect of IL-1 $\beta$ and Fn-fs on their production. IL-1 $\beta$ and Fn-fs increased the transcript and protein of ADAMTS-4 in HD- and OA-SF compared with the untreated cells (Figure 4, A and B). A significant increase in ADAMTS-5 transcript and protein was detected in OA-SF for both stimuli, whereas in HD stimulation was only observed in the protein after treatment with IL-1 $\beta$ (Figure 4, C and D). Altogether, these results show that IL-1 $\beta$ increased ADAMTS 4 and 5 in HD- and OA-SF, whereas Fn-fs showed more specific effects in OA, resulting in a significant augment of ADAMTS-4 in both, and in a restricted stimulation of ADAMTS-5 production in OA-SF.

\section{Aggrecanase Activity in SF and GAGs Release in Cartilage-SF Co-Cultures}

ADAMTS 4 and 5 cleave aggrecan within the interglobular domain at the Glu-373 and Ala-374 bond. $^{21,22}$ Thus, we assessed the ability of ADAMTS 4 and 5 produced by cultured SF to this cleavage by measuring aggrecanase activation and the ARGSVL-peptide-s released in culture supernatants by means of ELISA.

Both the constitutive aggrecanase activity (Figure 4E) and the derived peptide (data not shown) were significantly greater
A

ADAMTS-4

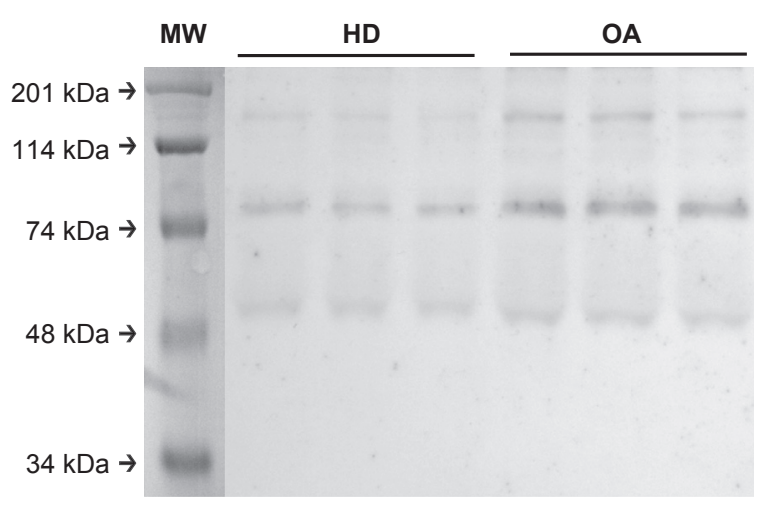

C

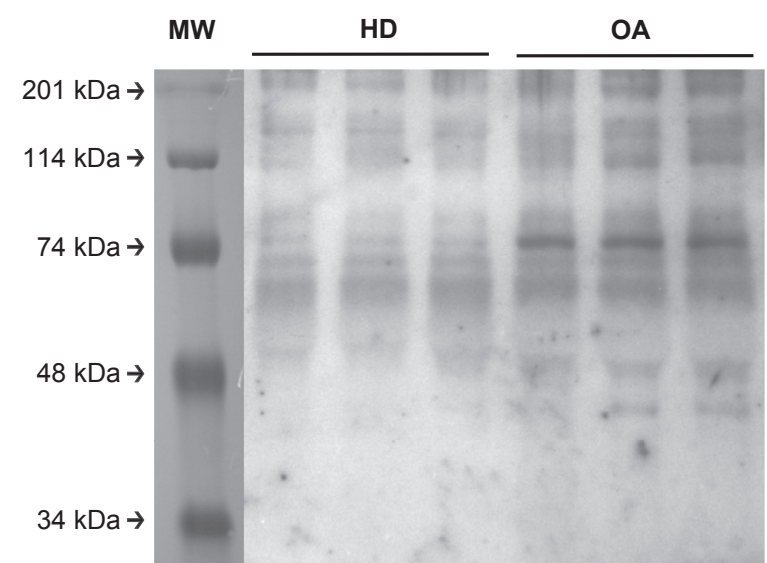

B

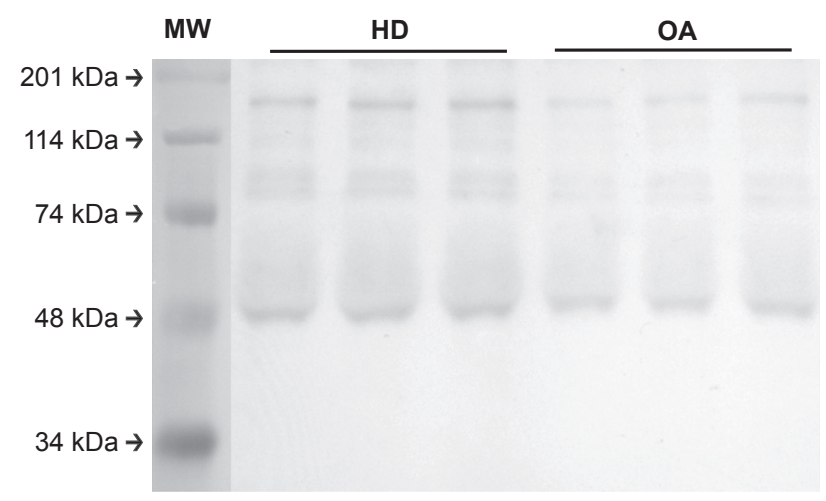

D

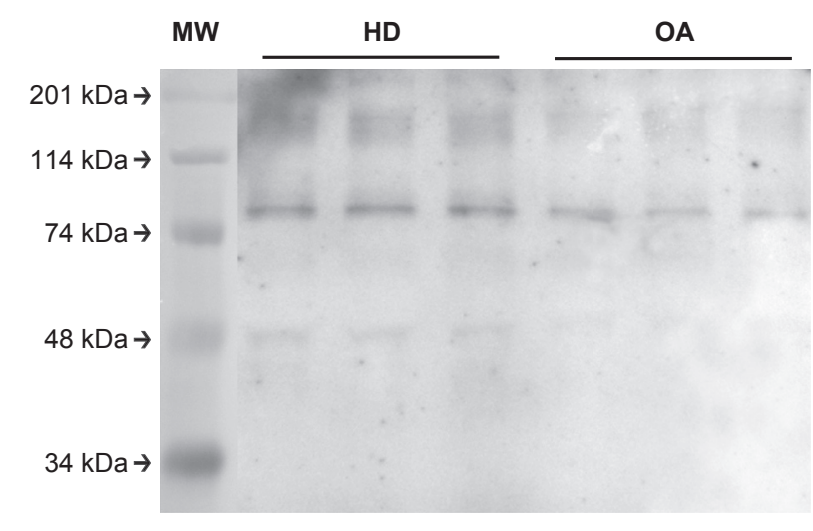

Figure 3 Western blot of ADAMTS in HD- and OA-SF. Presence of ADAMTS-4 (A), ADAMTS-5 (B), ADAMTS-7 (C), and ADAMTS-12 (D) was detected by Western blot in untreated HD- and OA-SF culture supernatants. Molecular weight (MW) markers on the polyvinylidene difluoride membrane are shown. $n=3$ (A-D, HD and OA). 
A

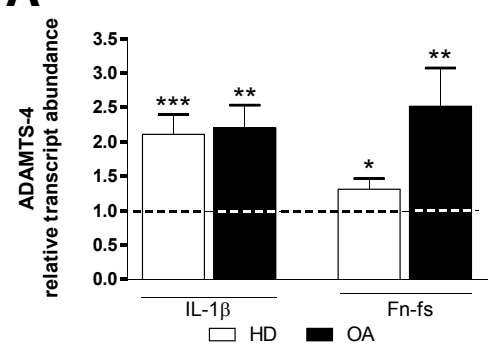

D

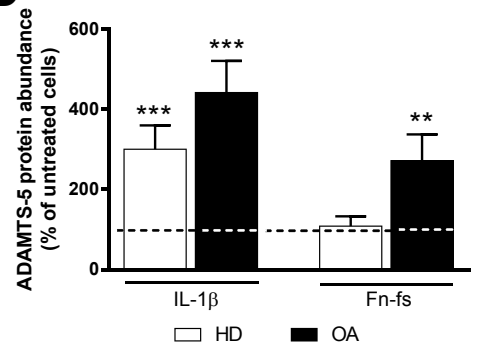

G

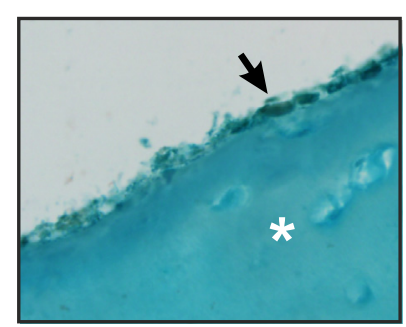

B

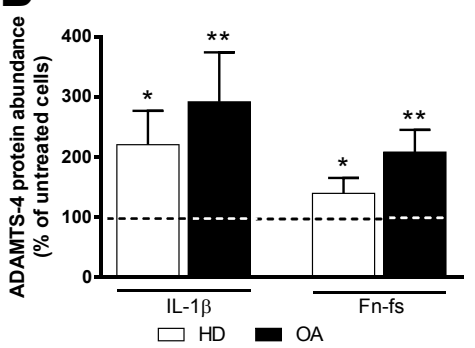

E

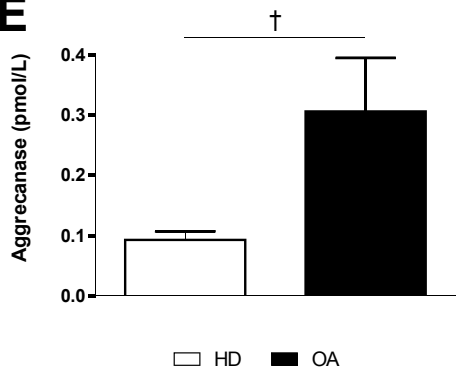

H

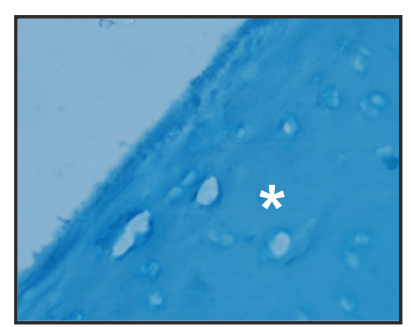

C

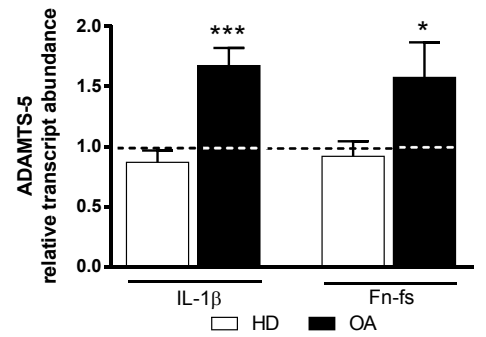

F

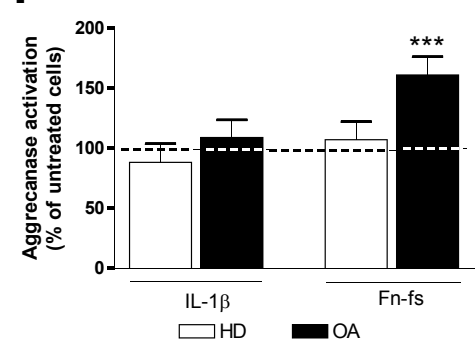

I

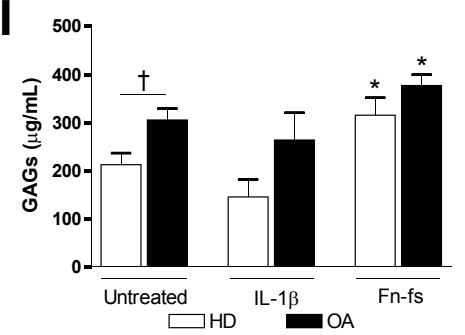

Figure 4 Induction of ADAMTS 4 and 5 by IL-1 $\beta$ or $45-\mathrm{kDa}$ Fn-fs in HD- and OA-SF. A and C: mRNA expression of ADAMTS 4 and 5 after treatment with IL-1 $\beta$ or $45-\mathrm{kDa}$ Fn-fs for 24 hours was measured by quantitative RT-PCR, normalized to $\beta$-actin, and presented as the relative quantification with respect to the untreated cells using the formula $2^{-\Delta \Delta \mathrm{Ct}}$ (described in Materials and Methods). Values are presented from triplicate determinations. B and D: Presence of ADAMTS 4 and 5 after treatment with IL-1 $\beta$ or $45-\mathrm{kDa}$ Fn-fs for 24 hours was determined by enzyme-linked immunosorbent assay (ELISA) in culture supernatants. Concentrations were calculated on the basis of the standard curve provided. Values are presented as the percentage of untreated cells (duplicate determinations). E and F: Aggrecanase activity in culture supernatants was measured by an Aggrecanase Activity ELISA kit, in untreated cells (E), and after treatment with IL-1 $\beta$ or $45-\mathrm{kDa}$ Fn-fs for 24 hours, presented as the percentage of untreated cells $(\mathbf{F})$. Values are from duplicate determinations. $\mathbf{G}$ and $\mathbf{H}$ : Representative histological sections of a cartilage explant (asterisks) in co-culture with OA-SF (arrow) and without SF (H). Alcian blue and Callejas's tricromic staining. I: Glycosaminoglycans (GAGs) in cartilage-SF co-culture supernatants after treatment with IL-1 $\beta$ or $45-k D a$ Fn-fs for 14 days were detected by a Blyscan Sulfated glycosaminoglycan assay. Values are from duplicate determinations. Dashed lines represent the untreated condition. Data are presented as means $\pm \operatorname{SEM}(\mathbf{A}-\mathbf{F}$ and $\mathbf{I}) . n=4(\mathbf{A}-\mathbf{F}, \mathrm{HD}) ; n=7(\mathbf{A}-\mathbf{D}, 0 \mathrm{~A}) ; n=5(\mathbf{E}$ and $\mathbf{F}, 0 \mathrm{~A}) ; n=3(\mathbf{I}, \mathrm{HD}$ and $0 \mathrm{~A}) .{ }^{*} P<0.05,{ }^{* *} P<0.01$, and ${ }^{* * *} P<0.001$ treatment versus untreated; ${ }^{\dagger} P<0.05 \mathrm{HD}$ versus $0 \mathrm{~A}$. Original magnification, $\times 20(\mathbf{G}-\mathbf{H})$.

in OA- compared to HD-SF. Fn-fs significantly increased aggrecanase activity (Figure $4 \mathrm{~F}$ ) and the derived peptide (data not shown) exclusively in OA-SF, whereas IL-1 $\beta$ did not induce any change. Interestingly, these results correlated with the Fn-fs induction of ADAMTS 4 and 5 in OA-SF.

The aggrecanase activity yields the generation of GAGs from the aggrecan in cartilage ECM. Hence, we next studied the potential capacity of $\mathrm{SF}$ to degrade cartilage by measuring GAG release in supernatants from cartilage-SF co-cultures. After 14 days of in vitro co-cultures, a monolayer of SF was observed exclusively on the cartilage surface (Figure 4G) that did not appear in the control (Figure $4 \mathrm{H})$. The effects of IL-1 $\beta$ and Fn-fs after 14 days of treatment were evaluated. The constitutive release of GAGs to the medium was significantly greater in OA-SF compared to HD-SF. IL-1 $\beta$ induced no change in GAGs levels, whereas
Fn-fs enhanced significantly the release of GAGs in both HD- and OA-SF (Figure 4I).

\section{Runx2 and $\beta$-Catenin Activation in SF}

Because Runx2 transcription factor and Wnt/ $\beta$-catenin signaling are involved in aggrecanase gene expression, ${ }^{16-18}$ and also seem to be implicated in the OA pathology, ${ }^{23,24}$ we decided to examine whether IL-1 $\beta$ or Fn-fs could alter the ADAMTS 4 and 5 expressions by the modulation of these factors.

Indeed, we found that both IL-1 $\beta$ and Fn-fs significantly induced nuclear activation of Runx 2 in HD and OA-SF (Figure 5A), consistent with its reduction in the cytoplasm after the stimulation with both mediators (Figure 5C). Nonetheless, the data were consistent with a role for Runx2 
A

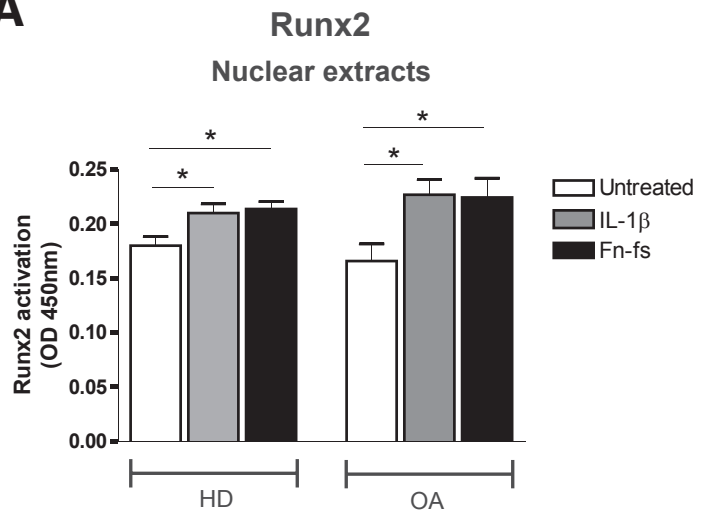

C

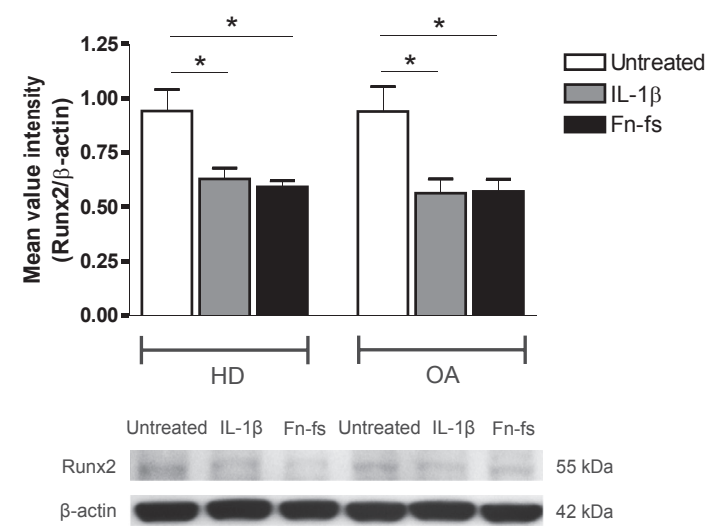

B

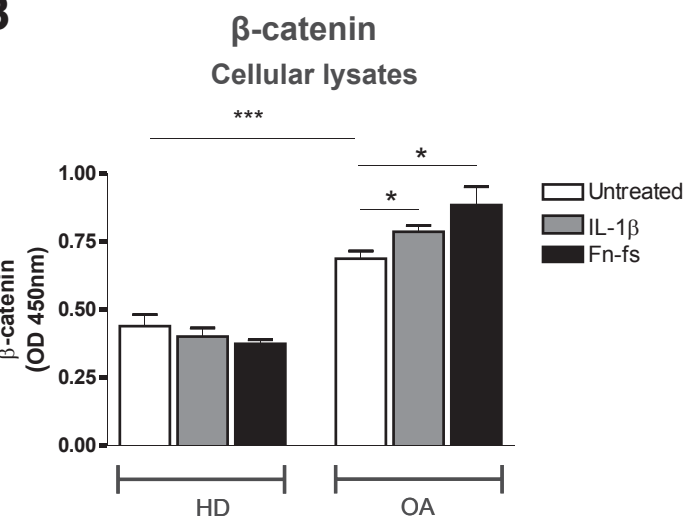

D

Cytoplasmic extracts
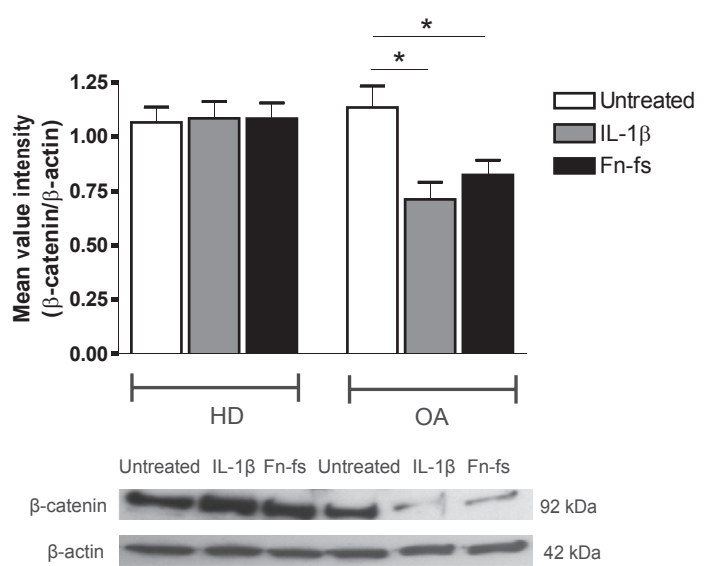

Figure 5 Activation of Runx2 and $\beta$-catenin by IL-1 $\beta$ or $45-k D a$ Fn-fs in HD- and OA-SF. A: Runx2 activation was measured in nuclear extracts after 60 minutes of treatment with IL-1 $\beta$ or 30 minutes of treatment with $45-\mathrm{kDa} F \mathrm{f}-\mathrm{fs}$, using a TransAM AML-3/Runx2 kit. Values are presented from duplicate determinations. B: $\beta$-Catenin was detected in cellular lysates after 60 minutes of treatment with IL- $1 \beta$ or $45-k D a$ Fn-fs, by enzyme-linked immunosorbent assay. Values are presented from duplicate determinations. C and D: Representative images of three independent experiments of the Western blots for Runx2 (C) and $\beta$-catenin (D) in cytoplasmic extracts. Protein bands were scanned and quantified with the Bio-Rad Quantity one program and presented. Values are presented as the ratio of mean value intensity normalized to $\beta$-actin of three independent experiments. Data are presented as means \pm SEM $(\mathbf{A}-\mathbf{D}) . n=4(\mathbf{A}$, $\mathrm{HD}$ and $\mathrm{OA}$, and $\mathbf{B}, \mathrm{HD}) ; n=5(\mathbf{B}, \mathrm{OA}) ; n=3(\mathbf{C}$ and $\mathbf{D}, \mathrm{HD}$ and $\mathrm{OA}) .{ }^{*} P<0.05 ;{ }^{* * *} P<0.001$.

in ADAMTS4 transcription because the transcript abundance with IL-1 $\beta$, on both HD and OA, was approximately twofold greater than in untreated controls. Furthermore, treatment with Fn-fs stimulated ADAMTS4 expression was approximately 2.5 -fold in OA cells and approximately 1.3 fold in HD cells. In contrast to ADAMTS-4, ADAMTS-5 expression showed no stimulation of HD-SF by either IL- $1 \beta$ or Fn-fs, but approximately a 1.6-fold increase by both IL-1 $\beta$ and Fn-fs in OA.

In the cytoplasm, $\beta$-catenin is regulated by interaction with a multiprotein complex that phosphorylates it to be degraded by proteasomes. On activation of Wnt signaling, non-phosphorylated $\beta$-catenin is transported to the nucleus, where it couples with the complex T-cell factor/lymphoidenhancing factor to initiate the transcription of ADAMTS 4 and 5 genes. ${ }^{25}$ We measured both $\beta$-catenin forms in cellular lysates of SF, where levels of phosphorylated $\beta$ catenin were undetectable by ELISA (data not shown). Thus, we measured the total $\beta$-catenin that mainly represented the active form.
The $\beta$-catenin content of whole cell lysates was approximately 1.5 -fold higher for untreated OA-SF than untreated HD. Moreover, $\beta$-catenin levels increased to approximately twofold after treatment with either IL-1 $\beta$ or Fn-fs exclusively in OA (Figure 5B), which correlated with the reduction observed in cytosplasmic extracts by Western blot (Figure 5D).

To better elucidate the implication of Runx 2 and $\beta$-catenin in the aggrecanases expression, we performed blockade experiments using inhibitors of two MAPK, ERK, and p38MAPK, implicated in the activation of Runx2, PD98059, and SB203580, respectively. We also used an inhibitor of Wnt/ $\beta$-catenin signaling, DDK-1. We showed that PD98059 significantly inhibited mRNA expression of ADAMTS-4 before treatment with IL-1 $\beta$ or Fn-fs, in HD- and OA-SF (Figure 6A). Moreover, PD98059 inhibited the expression of ADAMTS-5 in OA-SF after both stimuli, whereas in HD this inhibition was observed only before stimulation with IL-1 $\beta$ (Figure 6B). On the other hand, SB203580 is involved in the decrease of ADAMTS-4 mRNA expression 
A

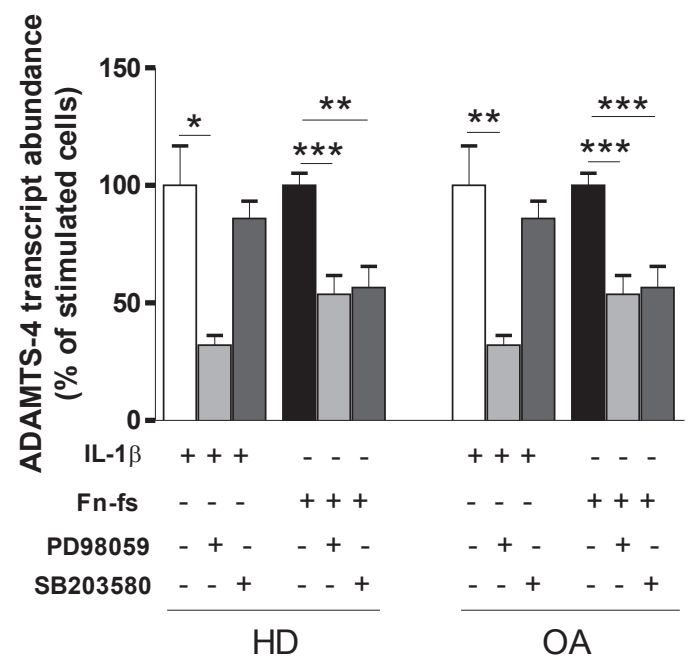

C

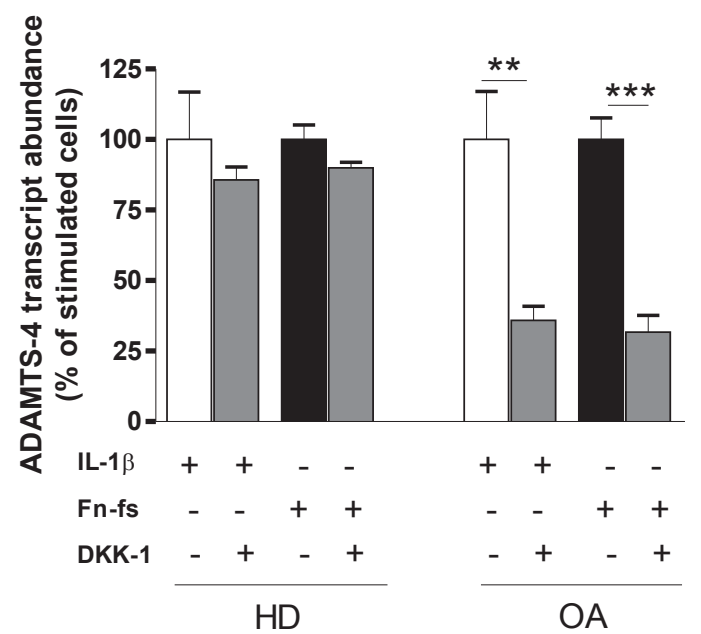

B

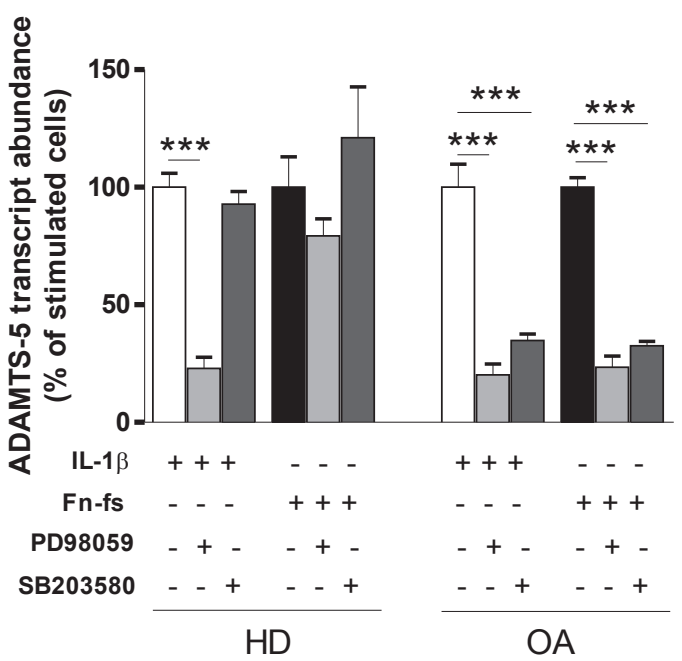

D

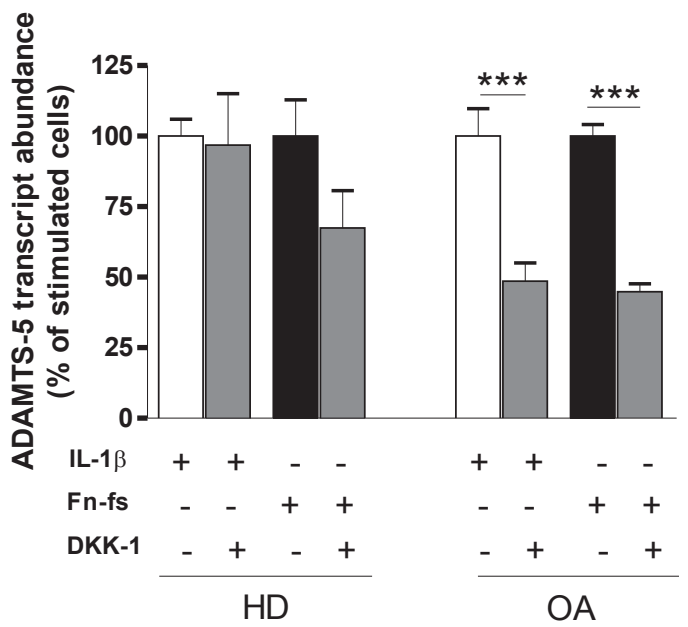

Figure 6 Blockade of Runx2 and Wnt/ $\beta$-catenin signaling. mRNA expression of ADAMTS 4 ( $A$ and $\mathbf{C}$ ) and 5 (B and D) was measured by quantitative RT-PCR, normalized to $\beta$-actin using the formula $2^{-\Delta \mathrm{Ct}}$, and presented as the percentage of stimulated cells, after 1 hour of treatment with inhibitors of two mitogenactivated protein kinases (MAPKs) implicated in the activation of Runx2, PD98059, a specific inhibitor of MAPK/extracellular signal-regulated kinase (ERK) kinases (MEK), responsible for the activation of ERK, and an inhibitor of p38-MAPK, SB203580 (A and B), or an inhibitor of Wnt signaling, DDK-1 (C and D), followed by treatment with IL-1 $\beta$ or $45-\mathrm{kDa}$ Fn-fs for 24 hours. Values are presented as means \pm SEM of triplicate determinations (A-D). $n=3(\mathbf{A}-\mathbf{D}, \mathrm{HD}$ and $0 \mathrm{~A}) .{ }^{*} P<0.05,{ }^{*} P<0.01$, and ${ }^{* * *} P<0.001$ inhibition versus stimulation.

stimulated by Fn-fs in both, HD- and OA-SF (Figure 6A). Regarding ADAMTS-5, SB203580 significantly inhibited its expression only in OA-SF, after both stimuli (Figure 6B). Moreover, the Wnt/ $\beta$-catenin inhibitor DKK-1 significantly inhibited the expression of both, ADAMTS 4 and 5, before treatment with IL-1 $\beta$ or Fn-fs, exclusively in OA-SF (Figure 6, C and D).

Induction of ADAMTS-7 and ADAMTS-12 by IL-1 $\beta$ or 45-kDa Fn-fs in SF, and COMP Production in Cartilage-SF Co-Cultures

We further studied the effects of IL- $1 \beta$ and Fn-fs on the ADAMTS involved in the degradation of COMP.
ADAMTS 7 and 12 share a C-terminal COMP/GEP-binding TSP domain. Their effects in OA are because of the association of this domain with COMP and its subsequent degradation. ${ }^{26}$ Significant increases in ADAMTS-7 transcript and protein were detected after IL-1 $\beta$ and Fn-fs stimulation (Figure 7, A and B). Regarding ADAMTS-12, we observed an increase of mRNA and protein induced by both stimuli exclusively in OA-SF (Figure 7, C and D).

The release of COMP and its degradative fragments was measured in the culture supernatants after 14 days of treatment with IL-1 $\beta$ and Fn-fs (Figure 7E). The constitutive release of COMP was significantly greater in OA-SF than in HD. Moreover, significant increases were detected after IL-1 $\beta$ and Fn-fs stimulation. 

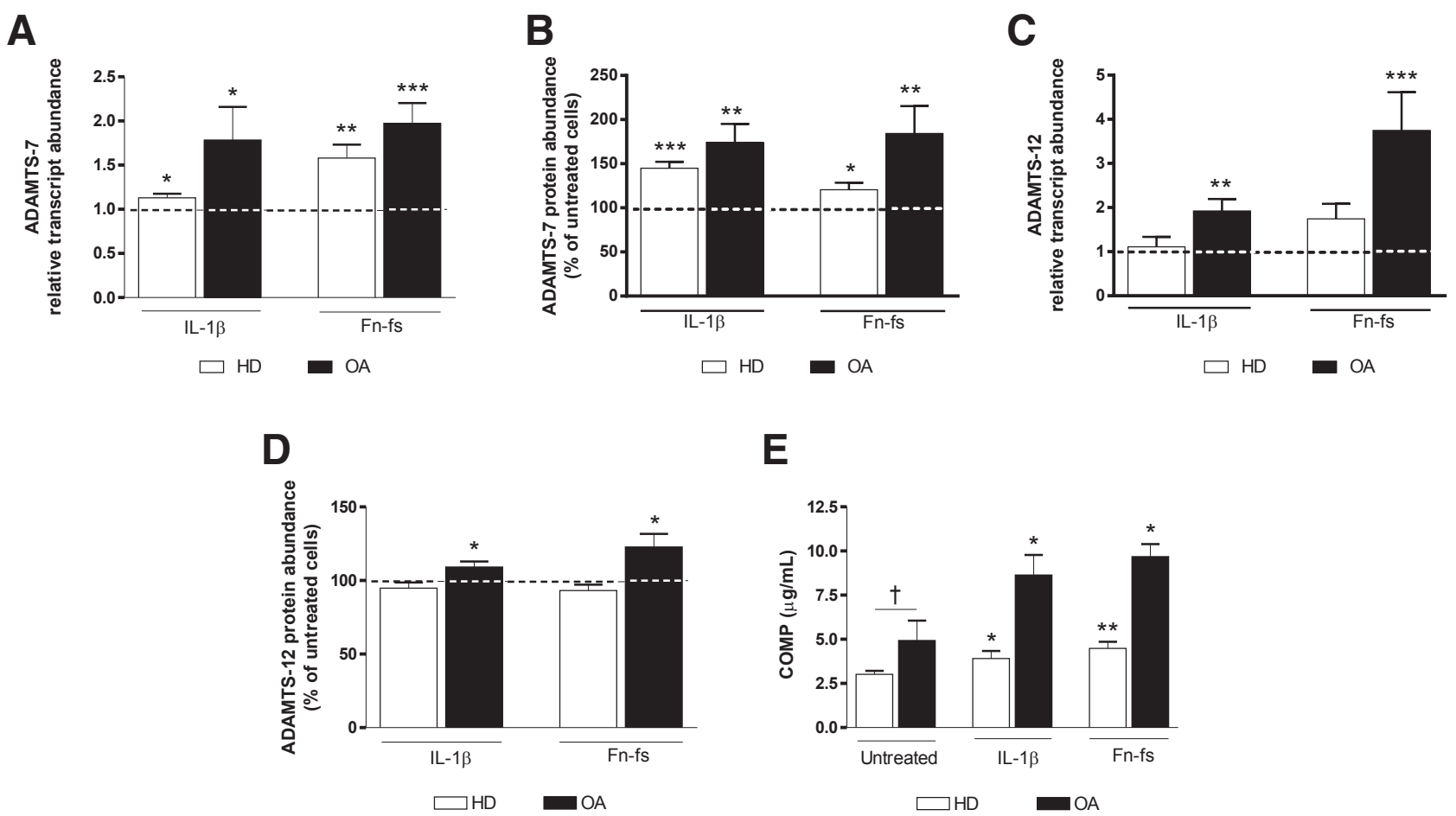

Figure 7 Induction of ADAMTS 7 and 12 by Fn-fs and IL-1 $\beta$ in HD- and OA-SF. A and C: mRNA expression of ADAMTS 7 and 12 after treatment with IL-1 $\beta$ or 45-kDa Fn-fs for 24 hours was measured by quantitative RT-PCR, normalized to $\beta$-actin, and presented as the relative quantification with respect to the untreated cells using the formula $2^{-\Delta \Delta \mathrm{Ct}}$ (described in Materials and Methods). Values are presented from triplicate determinations. B and D: Presence of ADAMTS 7 and 12 treatment with IL-1 $\beta$ or $45-\mathrm{kDa}$ Fn-fs for 24 hours was determined by enzyme-linked immunosorbent assay in culture supernatants. Values are presented as the percentage of untreated cells (duplicate determinations). E: Cartilage oligomeric matrix protein (COMP) in cartilage-SF co-culture supernatants after treatment with IL-1 $\beta$ or $45-\mathrm{kDa}$ Fn-fs for 14 days was detected by a Quantikine human COMP immunoassay. Values are presented from triplicate determinations. Dashed lines represent the untreated condition. Data are given as means $\pm \mathrm{SEM}(\mathbf{A}-\mathbf{E}) . n=4(\mathbf{A}-\mathbf{D}, \mathrm{HD}) ; n=7(\mathbf{A}-\mathbf{D}, 0 \mathrm{~A}) ; n=3$ (E, HD and $0 \mathrm{~A}) .{ }^{*} P<0.05,{ }^{* *} P<0.01$, and ${ }^{* * *} P<0.001$ treatment versus untreated; ${ }^{\dagger} P<0.05 \mathrm{HD}$ versus $0 \mathrm{~A}$.

\section{Discussion}

ADAMTS metalloproteinases play key roles in cartilage homeostasis and in the pathogenesis of OA, where the disruption of this balance, in favor of proteolysis, leads to a pathological cartilage destruction. ${ }^{3}$ ADAMTS 4, 5, 7, and 12 have been implicated in the breakdown of cartilage in $\mathrm{OA},{ }^{27,28}$ ADAMTS 4 and 5 degrading aggrecan, and ADAMTS 7 and 12 degrading COMP. Although their functions are well understood in cartilage, few studies have addressed the contribution of SF to their expression and release.

We showed that ADAMTS-5 was the most expressed in both HD- and OA-SF. Although ADAMTS4 gene expression was higher in OA than in HD, ADAMTS-5 mRNA expression was similar in both. Despite the fact that the levels of ADAMTS-5 are higher, differences in the ADAMTS-4 expression between HD and OA-SF seem to indicate its involvement in the OA pathology. However, lower levels of ADAMTS 4 and 5 have been reported in OA cartilage and synovium compared with non-OA tissues. ${ }^{29}$ Nevertheless, this discrepancy could be explained by the fact that whole synovium was used in their experiments. In addition, discordant data in the constitutive mRNA expression of ADAMTS 4 and 5 in non-OA and OA cartilage have been described, ${ }^{10}$ likely because of the different stages of the disease.

The complexity of ADAMTS regulation, by both pretranscriptional and post-transcriptional mechanisms, which ultimately determine the levels of secreted enzymes, has been reported. ${ }^{10}$ In this study, we demonstrated that both HD- and OA-SF release aggrecanases. Western blot analysis of ADAMTS 4 and 5 in SF culture supernatants revealed the presence of the active forms of both enzymes, between 48 and $74 \mathrm{kDa}$, similar to those reported in OA synovial fluids. ${ }^{30}$ We also observed a major band between 74 and $114 \mathrm{kDa}$, which has been described as the ADAMTS-4 proenzyme, in cartilage from OA patients, ${ }^{28}$ as well as in SF. ${ }^{9}$ Regarding ADAMTS-5, other authors have also reported a 70-kDa form of ADAMTS-5 in cartilage, as well as in SF from OA patients, ${ }^{9,28}$ that could represent different degradation fragments.

We reported that the aggrecanase activity and GAGs release are constitutively greater in OA than in HD. By contrast, no differences in the expression of aggrecanases between non-OA and OA synovium have been reported, ${ }^{9,31}$ with no data about their activity. Our results showed that IL-1 $\beta$ increased ADAMTS 4 and 5, with no impact in the 
aggrecanase activity or GAGs production. Because the protein abundance of ADAMTS-4 is apparently influenced by the transcript, in both HD and OA, whereas the protein abundance of ADAMTS- 5 after stimulation with IL-1 $\beta$ in HD does not correlate with the transcript, it seems likely that this ADAMTS is not the main aggrecanase. This fact corroborates other studies, which indicate that ADAMTS-5 mRNA levels do not correlate with the OA progression in chondrocytes, because of post-translational regulations. ${ }^{32} \mathrm{~A}$ recent study reported that IL-1 $\beta$ induced expression of ADAMTS-4 in SF but did not measure the activity. ${ }^{33}$ At the functional level, our results are in agreement with previous findings showing that ADAMTS 4 and 5 are not regulated by this cytokine in SF. ${ }^{9,12,31}$ The role of IL- $1 \beta$ in OA is controversial, and the implication of other mediators, as cartilage ECM degradation products, seems to be more relevant in the pathology. ${ }^{12}$ Despite that, the study of IL- $1 \beta$, as a pleiotropic proinflammatory cytokine, also contributes to the knowledge of the mechanism involved in the disease. In addition, because synovial inflammation intensity is greater in OA initial stages, ${ }^{4}$ the lack of effects observed in the aggrecanase activity after IL- $1 \beta$ stimulation could be explained by the advanced disease state of our patients. Although, after stimulation with IL-1 $\beta$ we observed effects in the levels of ADAMTS 4 and 5, they were not significant enough to promote their activity.

Articular cartilage matrix proteins are degraded in OA, resulting in the production of fragments with proinflammatory properties, including those of fibronectin. ${ }^{34,35}$ In chondrocytes, it has been described that the N-terminal Fnfs $45 \mathrm{kDa}$ induces matrix metalloproteinases synthesis and aggrecan degradation ${ }^{13,14,36}$; however, there are no data about its action in other joint cells. Herein, we describe for the first time the aggrecanases induction by Fn-fs in SF. Consistent with these previous reports, we noticed that Fn-fs produced a more specific effect in OA-SF, increasing aggrecanases production and activity, as well as cartilage degradation, evaluated by GAGs release from SF-cartilage co-cultures. ${ }^{14,36}$ Because ADAMTS-4 was also increased by Fn-fs in the co-cultures with HD- and OA-SF, these results again point to its main contribution to the aggrecan degradation. The integrin $\alpha 5 \beta 1$ is one of the main receptors implicated in the function of fibronectin, being involved in the cartilage proteoglycan degradation induced by the $45-\mathrm{kDa}$ Fn-fs. ${ }^{37,38}$ a $5 \beta 1$ integrins are also expressed in RA$\mathrm{SF}$, showing a significant increase compared with normal $\mathrm{SF}^{39}$ Thus, SF from OA patients would synthetize aggrecanases after $45-\mathrm{kDa}$ Fn-fs stimulation through integrins engagement. Our data suggest that in stages of SF hyperplasia in OA, Fn-fs could establish a feedback loop contributing to the maintenance of cartilage erosion.

Runx 2 can be predicted to promote ADAMTS 4 and 5 transcription. ${ }^{16,17} \mathrm{~A}$ recent study implicated it in the expression of ADAMTS 7 and 12 in OA cartilage also. ${ }^{24} \mathrm{On}$ the other hand, $\mathrm{Wnt} / \beta$-catenin signaling is a potent stimulator of chondrocyte matrix catabolic action triggering joint destruction, which also regulates aggrecanases expression. ${ }^{18}$ Both signaling pathways seem to play important roles in the OA pathophysiology. ${ }^{40-43}$ We showed that IL- $1 \beta$ and Fn-fs induced Runx2 in HD- and OA-SF. Moreover, ERK- and p38-MAPK are implicated in the activation of Runx2 transcription factor. ${ }^{44-46}$ Blockade experiments showed that ERK-MAPK is involved in the expression of ADAMTS 4 and 5 , stimulated by IL-1 $\beta$ or Fn-fs, in OA-SF. As both stimuli also induced the activation of Runx2 and the expression of both aggrecanases in these cells, we can conclude that ERK-MAPK signaling through Runx2 is involved in the expression of ADAMTS 4 and 5 in OA. ERK-MAPK also regulates the expression of ADAMTS-4 after both stimuli in HD-SF, in correlation with the induction of ADAMTS-4 transcript and protein, as well as with the activation of Runx 2 . These data suggest that IL-1 $\beta$ and Fn-fs could control ADAMTS-4 transcription via ERKMAPK and Runx2 also in SF from HD, by contrast to ADAMTS-5. On the other hand, p38-MAPK is only implicated in the expression of ADAMTS-4 after Fn-fs induction in HD- and OA-FLS, as well as in the induction of ADAMTS- 5 exclusively in OA-SF by both stimuli.

Of interest, we detected $\beta$-catenin induction exclusively in OA-SF, which also correlates with the restricted inhibition of Wnt signaling in OA-SF, mediated by DKK-1 before stimulation by both, IL-1 $\beta$ or $45-\mathrm{kDa}$ Fn-fs, pointing to the implication of this signaling pathway in the OA pathology. These results are in agreement with the higher expression of Wnt responsive genes, such as WISPI, in $\mathrm{OA},{ }^{47}$ which has been associated with a profibrotic and antichondrogenic OA-like phenotype. ${ }^{48}$ This interpretation is further supported by the finding that the Wnt7a/ $/$-catenin pathway promotes proteasomal degradation of Sox 9 , thereby blocking expression of chondrogenic genes. ${ }^{49}$

These results could shed some light on the understanding of how synovitis is triggered in OA, which is an issue under exploration. The disruption of the articular cartilage matrix is the most differencing feature in OA, and the resulting fragments of ECM catabolism have been associated with inflammation through the triggering of Toll-like receptor signaling pathways. Both IL- $1 \beta$ and Fn-fs induced the expression of Toll-like receptor 2 in chondrocytes. ${ }^{50}$ Thus, in addition to integrins, Fn-fs, as an endogenous ligand of Toll-like receptors 2 and 4, could act through its engagement, given that both receptors are present in SF. ${ }^{48,51}$ Moreover, both stimuli induce the activation of MAPK signaling, implicated in the activation of Runx 2 and other transcription factors, such as the NF- $\kappa \mathrm{B},{ }^{13-15,46,52}$ which also induce the expression of Wnt. ${ }^{53,54}$ Therefore, we can hypothesize that IL-1 $\beta$ and $45-\mathrm{kDa}$ Fn-fs induce aggrecanases expression by activation of the transcription factor Runx2, mainly through the ERK-MAPK signaling. Moreover, these stimuli specifically induce the Wnt/ $\beta$-catenin signaling in $\mathrm{OA}-\mathrm{SF}$, with the consequent therapeutic value.

ADAMTS 7 and 12 have been detected mainly in cartilage, with an up-regulated expression of their transcripts in 
OA compared with HD. Regarding other joint tissues, ADAMTS 7 and 12 mRNA expression have been previously described in whole synovium, with similar levels in $\mathrm{OA}$ and HD. ${ }^{29}$ In the present study, we show, for the first time, that isolated SF express and release ADAMTS 7 and 12. ADAMTS-7 transcripts were higher in OA- than in HDSF, whereas expression of ADAMTS-12 was similar in both. The higher expression of ADAMTS-7 in OA compared with HD also indicates the contribution of this ADAMTS in the OA pathology.

Our results are the first to describe the presence of ADAMTS 7 and 12 proteins in SF. Western blot of ADAMTS-7 showed a band between 114 and $201 \mathrm{kDa}$, similar to the active form previously described in a human kidney cell line. ${ }^{5,56}$ Regarding ADAMTS-12, we also reported a band between 114 and $201 \mathrm{kDa}$, equivalent to that previously reported in the same human line, ${ }^{33}$ and a smaller band between 74 and $114 \mathrm{kDa}$ that could represent the C-terminal fragment containing the TSP-1 repeats. ${ }^{57,58}$

As ADAMTS 7 and 12 are involved in the breakdown of arthritic articular cartilage, ${ }^{56,59}$ SFs represent other source of both metalloproteinases that would contribute to the maintenance of the cartilage damage. In this sense, COMP fragments have been identified in cartilage, synovial fluid, and serum from OA and rheumatoid arthritis patients. Moreover, increased levels of COMP in synovial fluid and serum are related to joint damage and progression in rheumatic diseases. ${ }^{59-61}$ Different joint tissues, such as bone, cartilage, synovium, and tendon, contain and express ADAMTS-7, which colocalizes with ADAMTS-12 and COMP in the cytoplasm of chondrocytes. ${ }^{62,63}$ Consistent with this, we reported herein that cartilage-OA-SF co-cultures constitutively release COMP to the medium at significantly higher levels than cartilage-HD-SF co-cultures.

Tumor necrosis factor- $\alpha$ and IL- $1 \beta$ increase ADAMTS 7 and 12 mRNA in cartilage explants, ${ }^{64}$ whereas ADAMTS- 12 is not induced in human fetal fibroblasts. ${ }^{57}$ We report, for the first time in SF, that IL-1 $\beta$ and Fn-fs promoted significant increases in ADAMTS-7 transcripts and protein levels, being greater in OA- than in HD-SF. Similarly, ADAMTS-12 transcripts and protein were stimulated by IL- $1 \beta$ and Fn-fs specifically in OA-SF. Interestingly, ADAMTS-12 was the only ADAMTS studied exclusively induced in OA-SF. Those facts are important for the development of combined therapies on the basis of the blockade of these ADAMTS. Emergent data indicate that ADAMTS-12 has multiple functions in the inflammatory response, ${ }^{64}$ angiogenesis, and apoptosis. ${ }^{65-67}$ Thus, further studies are needed to clarify whether ADAMTS-12 is also involved in those functions during OA development.

Our results showed that the stimulation of COMP release by IL- $1 \beta$ and Fn-fs was greater in OA-SF. Because specific antibodies against ADAMTS 7 and 12 inhibited the tumor necrosis factor- $\alpha$ - or IL-1 $\beta$-induced COMP degradation in the cartilage of OA patients, ${ }^{68}$ this increased release of COMP after IL-1 $\beta$ or Fn-fs treatment could be ascribed to the increased expression of both ADAMTS. Because, by contrast to ADAMTS-12, IL-1 $\beta$ and Fn-fs also induced ADAMTS-7 in HD-SF, COMP degradation may be mainly attributed to its action. Our data suggest that in the late phase of OA, IL-1 $\beta$ and Fn-fs may contribute to the damage of noncollagenous components of the ECM by increasing ADAMTS 7 and 12.

Our study presents two potential limitations. The in vitro model of cartilage-SF co-culture uses dead cartilage; thus, whether the same results would be obtained with SF adhered to live human OA cartilage is unknown. Besides, the medium used for all cell cultures was DMEM containing high glucose concentration. However, given that all treatments were performed with the same medium, this condition would not invalidate our results.

Overall, our data indicate that SF provides aggrecanases, ADAMTS-7, and ADAMTS-12 that contribute to the chronicity and destruction of OA joint. Although both IL- $1 \beta$ and Fn-fs have been described as mediators of cartilage degradation in OA, our findings indicate that despite the contribution of both mediators, they are Fn-fs rather than IL-1 $\beta$, which plays the major pathological role, in agreement with recent studies. ${ }^{12}$ We showed that constitutive levels of ADAMTS-4, one of the main aggrecanases in cartilage destruction, were higher in OA than in HD. Interestingly, the higher levels of ADAMTS-7 in OA compared to HD, as well as the regulation of ADAMTS- 12 by IL- $1 \beta$ and Fn-fs exclusively in SF from OA patients, suggest their potential as new therapeutic targets for the treatment of the disease. Altogether, our results point to an important contribution of $\mathrm{SF}$, providing the biochemical tools, to the chronicity and destruction of the osteoarthritic affected joint.

\section{Acknowledgments}

We thank all patients and the collaborating clinicians for their participation in this study and Dr. Mary B. Goldring (Hospital for Special Surgery, New York, NY) for contributions to the final manuscript.

\section{References}

1. Brooks P: Inflammation as an important feature of osteoarthritis. Bull World Health Organ 2003, 81:689-690

2. Goldring MB, Goldring SR: Osteoarthritis. J Cell Physiol 2007, 213: 626-634

3. Goldring MB, Marcu KB: Cartilage homeostasis in health and rheumatic diseases. Arthritis Res Ther 2009, 11:224

4. Benito MJ, Veale DJ, FitzGerald O, van den Berg WB, Bresnihan B: Synovial tissue inflammation in early and late osteoarthritis. Ann Rheum Dis 2005, 64:1263-1267

5. Lefevre S, Meier FM, Neumann E, Muller-Ladner U: Role of synovial fibroblasts in rheumatoid arthritis. Curr Pharm Des 2015, 21: $130-141$

6. Bondeson J, Blom AB, Wainwright S, Hughes C, Caterson B, van den Berg WB: The role of synovial macrophages and macrophageproduced mediators in driving inflammatory and destructive responses in osteoarthritis. Arthritis Rheum 2010, 62:647-657 
7. Sutton S, Clutterbuck A, Harris P, Gent T, Freeman S, Foster N, Barrett-Jolley R, Mobasheri A: The contribution of the synovium, synovial derived inflammatory cytokines and neuropeptides to the pathogenesis of osteoarthritis. Vet J 2009, 179:10-24

8. Lin EA, Liu CJ: The role of ADAMTSs in arthritis. Protein Cell 2010, 1:33-47

9. Yamanishi Y, Boyle DL, Clark M, Maki RA, Tortorella MD, Arner EC, Firestein GS: Expression and regulation of aggrecanase in arthritis: the role of TGF-beta. J Immunol 2002, 168:1405-1412

10. Fosang AJ, Rogerson FM: Identifying the human aggrecanase. Osteoarthritis Cartilage 2010, 18:1109-1116

11. Acharya C, Yik JH, Kishore A, Van Dinh V, Di Cesare PE, Haudenschild DR: Cartilage oligomeric matrix protein and its binding partners in the cartilage extracellular matrix: interaction, regulation and role in chondrogenesis. Matrix Biol 2014, 37:102-111

12. Sandy JD, Chan DD, Trevino RL, Wimmer MA, Plaas A: Human genome-wide expression analysis reorients the study of inflammatory mediators and biomechanics in osteoarthritis. Osteoarthritis Cartilage 2015, 23:1939-1945

13. Ding L, Guo D, Homandberg GA: Fibronectin fragments mediate matrix metalloproteinase upregulation and cartilage damage through proline rich tyrosine kinase 2, c-src, NF-kappaB and protein kinase Cdelta. Osteoarthritis Cartilage 2009, 17:1385-1392

14. Ding L, Guo D, Homandberg GA: The cartilage chondrolytic mechanism of fibronectin fragments involves MAP kinases: comparison of three fragments and native fibronectin. Osteoarthritis Cartilage 2008, 16:1253-1262

15. Yasuda T: Cartilage destruction by matrix degradation products. Mod Rheumatol 2006, 16:197-205

16. Thirunavukkarasu K, Pei Y, Moore TL, Wang H, Yu XP, Geiser AG, Chandrasekhar S: Regulation of the human ADAMTS-4 promoter by transcription factors and cytokines. Biochem Biophys Res Comm 2006, 345:197-204

17. Thirunavukkarasu K, Pei Y, Wei T: Characterization of the human ADAMTS-5 (aggrecanase-2) gene promoter. Mol Biol Rep 2007, 34: 225-231

18. Yuasa T, Otani T, Koike T, Iwamoto M, Enomoto-Iwamoto M: Wnt/B-catenin signaling stimulates matrix catabolic genes and activity in articular chondrocytes: its possible role in joint degeneration. Lab Invest 2008, 88:264-274

19. Juarranz Y, Gutierrez-Canas I, Santiago B, Carrion M, Pablos JL, Gomariz RP: Differential expression of vasoactive intestinal peptide and its functional receptors in human osteoarthritic and rheumatoid synovial fibroblasts. Arthritis Rheum 2008, 58:1086-1095

20. Pretzel D, Pohlers D, Weinert S, Kinne RW: In vitro model for the analysis of synovial fibroblast-mediated degradation of intact cartilage. Arthritis Res Ther 2009, 11:R25

21. Huang K, Wu LD: Aggrecanase and aggrecan degradation in osteoarthritis: a review. J Int Med Res 2008, 36:1149-1160

22. Kelwick R, Desanlis I, Wheeler GN, Edwards DR: The ADAMTS (a disintegrin and metalloproteinase with thrombospondin motifs) family. Genome Biol 2015, 16:113

23. Alcaraz MJ, Megias J, Garcia-Arnandis I, Clerigues V, Guillen MI: New molecular targets for the treatment of osteoarthritis. Biochem Pharmacol 2010, 80:13-21

24. Ji Q, Xu X, Xu Y, Fan Z, Kang L, Li L, Liang Y, Guo J, Hong T, Li Z, Zhang Q, Ye Q, Wang Y: miR-105/Runx2 axis mediates FGF2induced ADAMTS expression in osteoarthritis cartilage. J Mol Med (Berl) 2016, 94:681-694

25. Thomas RS, Clarke AR, Duance VC, Blain EJ: Effects of Wnt3A and mechanical load on cartilage chondrocyte homeostasis. Arthritis Res Ther 2011, 13:R203

26. Lin EA, Liu CJ: The emerging roles of ADAMTS-7 and ADAMTS12 matrix metalloproteinases. Rheumatol Res Rev 2009, 1:121-131

27. Zhang Q, Huang M, Wang X, Xu X, Ni M, Wang Y: Negative effects of ADAMTS-7 and ADAMTS-12 on endplate cartilage differentiation. J Orthop Res 2012, 30:1238-1243
28. Malfait AM, Liu RQ, Ijiri K, Komiya S, Tortorella MD: Inhibition of ADAM-TS4 and ADAM-TS5 prevents aggrecan degradation in osteoarthritic cartilage. J Biol Chem 2002, 277:22201-22208

29. Davidson RK, Waters JG, Kevorkian L, Darrah C, Cooper A, Donell ST, Clark IM: Expression profiling of metalloproteinases and their inhibitors in synovium and cartilage. Arthritis Res Ther 2006, 8: R124

30. Zhang E, Yan X, Zhang M, Chang X, Bai Z, He Y, Yuan Z: Aggrecanases in the human synovial fluid at different stages of osteoarthritis. Clin Rheumatol 2013, 32:797-803

31. Vankemmelbeke MN, Holen I, Wilson AG, Ilic MZ, Handley CJ, Kelner GS, Clark M, Liu C, Maki RA, Burnett D, Buttle DJ: Expression and activity of ADAMTS-5 in synovium. Eur J Biochem 2001, 268:1259-1268

32. Yamamoto K, Troeberg L, Scilabra SD, Pelosi M, Murphy CL, Strickland DK, Nagase H: LRP-1-mediated endocytosis regulates extracellular activity of ADAMTS-5 in articular cartilage. FASEB J 2013, 27:511-521

33. Kataoka Y, Ariyoshi W, Okinaga T, Kaneuji T, Mitsugi S, Takahashi T, Nishihara T: Mechanisms involved in suppression of ADAMTS4 expression in synoviocytes by high molecular weight hyaluronic acid. Biochem Biophys Res Commun 2013, 432:580-585

34. Homandberg GA, Wen C, Hui F: Cartilage damaging activities of fibronectin fragments derived from cartilage and synovial fluid. Osteoarthritis Cartilage 1998, 6:231-244

35. Zack MD, Arner EC, Anglin CP, Alston JT, Malfait AM, Tortorella MD: Identification of fibronectin neoepitopes present in human osteoarthritic cartilage. Arthritis Rheum 2006, 54:2912-2922

36. Stanton H, Ung L, Fosang AJ: The $45 \mathrm{kDa}$ collagen-binding fragment of fibronectin induces matrix metalloproteinase- 13 synthesis by chondrocytes and aggrecan degradation by aggrecanases. Biochem J 2002, 364:181-190

37. Homandberg GA, Costa V, Ummadi V, Pichika R: Antisense oligonucleotides to the integrin receptor subunit alpha(5) decrease fibronectin fragment mediated cartilage chondrolysis. Osteoarthritis Cartilage 2002, 10:381-393

38. Homandberg GA, Costa V, Wen C: Fibronectin fragments active in chondrocytic chondrolysis can be chemically cross-linked to the alpha5 integrin receptor subunit. Osteoarthritis Cartilage 2002, 10: 938-949

39. Rinaldi N, Schwarz-Eywill M, Weis D, Leppelmann-Jansen P, Lukoschek M, Keilholz U, Barth TF: Increased expression of integrins on fibroblast-like synoviocytes from rheumatoid arthritis in vitro correlates with enhanced binding to extracellular matrix proteins. Ann Rheum Dis 1997, 56:45-51

40. Luyten FP, Tylzanowski P, Lories RJ: Wnt signaling and osteoarthritis. Bone 2009, 44:522-527

41. Tornero-Esteban P, Peralta-Sastre A, Herranz E, RodriguezRodriguez L, Mucientes A, Abasolo L, Marco F, FernandezGutierrez B, Lamas JR: Altered expression of Wnt signaling pathway components in osteogenesis of mesenchymal stem cells in osteoarthritis patients. PLoS One 2015, 10:e0137170

42. Saito T, Nishida K, Furumatsu T, Yoshida A, Ozawa M, Ozaki T: Histone deacetylase inhibitors suppress mechanical stress-induced expression of RUNX-2 and ADAMTS-5 through the inhibition of the MAPK signaling pathway in cultured human chondrocytes. Osteoarthritis Cartilage 2013, 21:165-174

43. Tetsunaga $T$, Nishida $K$, Furumatsu $T$, Naruse $K$, Hirohata $S$, Yoshida A, Saito T, Ozaki T: Regulation of mechanical stressinduced MMP-13 and ADAMTS-5 expression by RUNX-2 transcriptional factor in SW1353 chondrocyte-like cells. Osteoarthritis Cartilage 2011, 19:222-232

44. Vimalraj S, Arumugam B, Miranda PJ, Selvamurugan N: Runx2: structure, function, and phosphorylation in osteoblast differentiation. Int J Biol Macromol 2015, 78:202-208

45. Huang YF, Lin JJ, Lin CH, Su Y, Hung SC: c-Jun N-terminal kinase 1 negatively regulates osteoblastic differentiation induced by BMP2 
via phosphorylation of Runx2 at Ser104. J Bone Miner Res 2012, 27: 1093-1105

46. Rasheed Z, Akhtar N, Haqqi TM: Pomegranate extract inhibits the interleukin-1beta-induced activation of MKK-3, p38alpha-MAPK and transcription factor RUNX-2 in human osteoarthritis chondrocytes. Arthritis Res Ther 2010, 12:R195

47. van den Bosch MH, Gleissl TA, Blom AB, van den Berg WB, van Lent PL, van der Kraan PM: Wnts talking with the TGF-beta superfamily: WISPers about modulation of osteoarthritis. Rheumatology (Oxford) 2015, [Epub ahead of print] doi:10.1093/rheumatology/ kev402

48. Gutierrez-Canas I, Juarranz Y, Santiago B, Arranz A, Martinez C, Galindo M, Paya M, Gomariz RP, Pablos JL: VIP down-regulates TLR4 expression and TLR4-mediated chemokine production in human rheumatoid synovial fibroblasts. Rheumatology (Oxford) 2006, 45:527-532

49. Jin EJ, Lee SY, Choi YA, Jung JC, Bang OS, Kang SS: BMP-2enhanced chondrogenesis involves p38 MAPK-mediated downregulation of Wnt-7a pathway. Mol Cells 2006, 22:353-359

50. Su SL, Tsai CD, Lee CH, Salter DM, Lee HS: Expression and regulation of Toll-like receptor 2 by IL-1beta and fibronectin fragments in human articular chondrocytes. Osteoarthritis Cartilage 2005, $13: 879-886$

51. You R, Zheng M, McKeown-Longo PJ: The first type III repeat in fibronectin activates an inflammatory pathway in dermal fibroblasts. J Biol Chem 2010, 285:36255-36259

52. Stylianou E, Saklatvala J: Interleukin-1. Int J Biochem Cell Biol 1998, 30:1075-1079

53. Lee JG, Heur M: Interleukin-1beta-induced Wnt5a enhances human corneal endothelial cell migration through regulation of $\mathrm{Cdc} 42$ and RhoA. Mol Cell Biol 2014, 34:3535-3545

54. Raymond M, Marchbank T, Moyer MP, Playford RJ, Sanderson IR, Kruidenier L: IL-1beta stimulation of CCD-18co myofibroblasts enhances repair of epithelial monolayers through Wnt-5a. Am J Physiol Gastrointest Liver Physiol 2012, 303:G1270-G1278

55. Bai XH, Wang DW, Kong L, Zhang Y, Luan Y, Kobayashi T, Kronenberg HM, Yu XP, Liu CJ: ADAMTS-7, a direct target of PTHrP, adversely regulates endochondral bone growth by associating with and inactivating GEP growth factor. Mol Cell Biol 2009, 29: 4201-4219

56. Bai XH, Wang DW, Luan Y, Yu XP, Liu CJ: Regulation of chondrocyte differentiation by ADAMTS-12 metalloproteinase depends on its enzymatic activity. Cell Mol Life Sci 2009, 66:667-680

57. Cal S, Arguelles JM, Fernandez PL, Lopez-Otin C: Identification, characterization, and intracellular processing of ADAM-TS12, a novel human disintegrin with a complex structural organization involving multiple thrombospondin-1 repeats. J Biol Chem 2001, 276:17932-17940

58. Beristain AG, Zhu H, Leung PC: Regulated expression of ADAMTS12 in human trophoblastic cells: a role for ADAMTS-12 in epithelial cell invasion? PLoS One 2011, 6:e18473

59. Liu CJ, Kong W, Xu K, Luan Y, Ilalov K, Sehgal B, Yu S, Howell RD, Di Cesare PE: ADAMTS-12 associates with and degrades cartilage oligomeric matrix protein. J Biol Chem 2006, 281:15800-15808

60. Liu CJ, Kong W, Ilalov K, Yu S, Xu K, Prazak L, Fajardo M, Sehgal B, Di Cesare PE: ADAMTS-7: a metalloproteinase that directly binds to and degrades cartilage oligomeric matrix protein. FASEB J 2006, 20:988-990

61. Andersson ML, Svensson B, Petersson IF, Hafstrom I, Albertsson K, Forslind K, Heinegard D, Saxne T: Early increase in serum-COMP is associated with joint damage progression over the first five years in patients with rheumatoid arthritis. BMC Musculoskelet Disord 2013, $14: 229$

62. Hedbom E, Antonsson P, Hjerpe A, Aeschlimann D, Paulsson M, Rosa-Pimentel E, Sommarin Y, Wendel M, Oldberg A, Heinegard D: Cartilage matrix proteins: an acidic oligomeric protein (COMP) detected only in cartilage. J Biol Chem 1992, 267:6132-6136

63. DiCesare P, Hauser N, Lehman D, Pasumarti S, Paulsson M: Cartilage oligomeric matrix protein (COMP) is an abundant component of tendon. FEBS Lett 1994, 354:237-240

64. Moncada-Pazos A, Obaya AJ, Llamazares M, Heljasvaara R, Suarez MF, Colado E, Noel A, Cal S, Lopez-Otin C: ADAMTS-12 metalloprotease is necessary for normal inflammatory response. J Biol Chem 2012, 287:39554-39563

65. Llamazares M, Obaya AJ, Moncada-Pazos A, Heljasvaara R, Espada J, Lopez-Otin C, Cal S: The ADAMTS12 metalloproteinase exhibits anti-tumorigenic properties through modulation of the Rasdependent ERK signalling pathway. J Cell Sci 2007, 120: $3544-3552$

66. Moncada-Pazos A, Obaya AJ, Fraga MF, Viloria CG, Capella G, Gausachs M, Esteller M, Lopez-Otin C, Cal S: The ADAMTS12 metalloprotease gene is epigenetically silenced in tumor cells and transcriptionally activated in the stroma during progression of colon cancer. J Cell Sci 2009, 122:2906-2913

67. Wei J, Richbourgh B, Jia T, Liu C: ADAMTS-12: a multifaced metalloproteinase in arthritis and inflammation. Mediators Inflamm 2014, 2014:649718

68. Luan Y, Kong L, Howell DR, Ilalov K, Fajardo M, Bai XH, Di Cesare PE, Goldring MB, Abramson SB, Liu CJ: Inhibition of ADAMTS-7 and ADAMTS-12 degradation of cartilage oligomeric matrix protein by alpha-2-macroglobulin. Osteoarthritis Cartilage 2008, 16:1413-1420 